\title{
SUPPORTING INFORNATION
}

\section{On-chip integration of energy-tunable quantum dots based single-photon sources via strain tuning of GaAs waveguides}

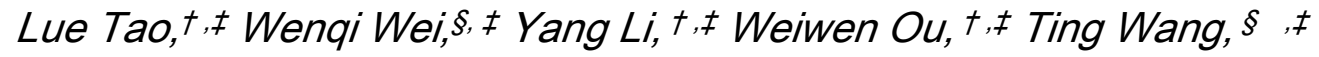

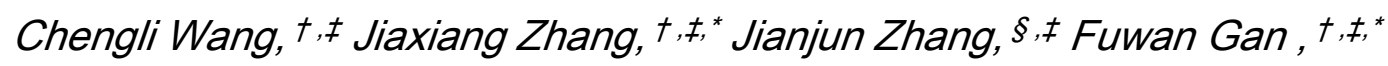
and Xin Out,, , *

$\dagger$ State Key Laboratory of Functional Materials for Informatics, Shanghai Institute of Microsystem and Information Technology, Chinese Academy of Sciences, Shanghai, 200092, China

$\S$ Beijing National Laboratory for Condensed Matter Physics, Institute of Physics, Chinese Academy of Sciences, Beijing 100190, China

$\star$ Center of Materials Science and Optoelectronics Engineering, University of Chinese Academy of Sciences, Beijing 100049, China 


\section{Sample structure and detailed fabrication process for the hybrid quantum photonic chip}

The investigated sample was grown by solid source molecular beam epitaxy (MBE) on a 2-inch semi-insulating GaAs (001) substrate with a thickness of $500 \mu \mathrm{m}$. It started with deposition of a $200 \mathrm{~nm}$ thick undoped GaAs buffer layer on the substrate at temperature of about $580{ }^{\circ} \mathrm{C}$. Then a layer of self-assembled InGaAs quantum dots (QDs) sandwiched in the middle of $300 \mathrm{~nm}$ thick GaAs layer was grown on top of a $100 \mathrm{~nm}$ thick $\mathrm{Al}_{0.75} \mathrm{Ga}_{0.25} \mathrm{As}$ sacrificial layer. Due to the inherent temperature gradient (about $10^{\circ} \mathrm{C}$ from the center to the edge), the QD density varied across the wafer and different density regions were identified by micro-photoluminescence ( $\mu$-PL) spatial mapping ${ }^{1}$. In the present work, a region of $5 \times 5 \mathrm{~mm}^{2}$ with QD density of $10^{7} \sim 10^{8} \mathrm{~cm}^{-2}$ was selected for the fabrication of the hybrid photonic structures.

The detailed fabrication process is depicted in Figure S1. First of all, a $300 \mathrm{~nm}$ thick photoresist ZEP520A was deposited on the as-grown GaAs substrate. Photonic structures including waveguides and grating couplers were patterned by using an electron-beam lithography tool (EBPG 5200). After developing in n-amylacetate for 1 min and rinsing in IPA for $10 \mathrm{~s}$, the GaAs photonic structures were etched in an RIEICP (SENTECH SI500) process by using chlorine-based chemistry with the ZEP520A mask. In the next step, rectangular patterns made of AZ5214 photoresist were defined by means of standard UV photolithography. The uncovered area was non-selectively etched in a sulfuric acid solution $\left(\mathrm{H}_{2} \mathrm{SO}_{4}: \mathrm{H}_{2} \mathrm{O}_{2}: \mathrm{H}_{2} \mathrm{O}=1: 8: 200\right)$ and the residual resist was cleaned by soaking the sample in acetone for $5 \mathrm{~min}$. Diluted hydrofluoric acid (HF $40 \%$ mixed 1:3 with water by volume) was then used to selectively remove the underneath $\mathrm{Al}_{0.75} \mathrm{Ga}_{0.25} \mathrm{As}$ sacrificial layer, leaving the GaAs nanomembranes with photonic structures unbound on top of the substrate. Since the GaAs has low mechanical stiffness, the HF etching led the GaAs nanomembranes to collapse and stick on the substrate due to an inter-solid adhesion. Figure $\mathbf{S} 1 \mathrm{k}$ shows a typical microscopic image of the nanomembranes on the substrate after the HF etching. In the final step, the freestanding GaAs nanomembranes were transferred onto a piezoelectric actuator (PMNPT) by a transfer-printing technique via a viscoelastic stamp (PDMS). In this technique, we used a thin layer of PMMA on the PMN-PT actuator as glue and then applied heat $\left(90{ }^{\circ} \mathrm{C}\right)$ and small pressure simultaneously to bond the GaAs nanomembranes with photonic structures onto the PMN-PT actuator. Figure $\mathbf{S} 1 \mathbf{j}$ shows a microscopic image of the fabricated hybrid quantum photonic chip. It is also worth noting that the yield for successful transferring of the GaAs photonic chip is rather high and about $80 \%$ of the processed GaAs photonic structures have been achieved experimentally. 


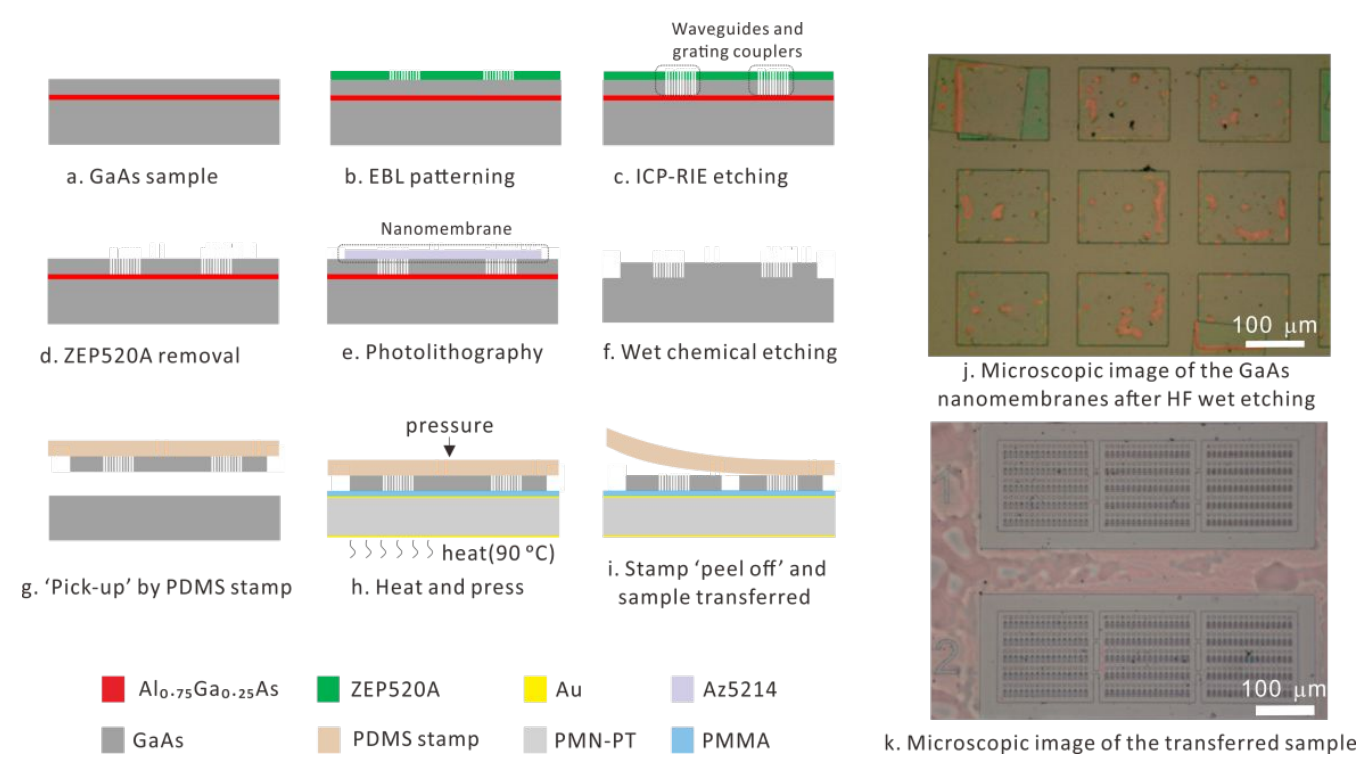

Figure S1. Process flow required for fabricating the hybrid quantum photonic chip. (a) - (d) EBL and RIE-ICP processes for patterning and dry etching of the photonic structures on the GaAs substrate. (e) - (f) Standard UV Photolithography and wet chemical etching for the fabrication of the free-standing GaAs nanomembranes. (g) (i) Dry transfer of the GaAs nanomembranes containing photonic structures onto the PMN-PT actuator via the PDMS viscoelastic stamp. (j) - (k) Microscopic images of the GaAs nanomembranes after HF etching and the transferred GaAs photonic chip on the PMN-PT substrate.

\section{Experimental methods}

We characterized the hybrid photonic chip in a home-built $\mu$-PL setup as shown in Figure $\mathbf{S} 2$. The sample was firstly mounted on a $x y z$ nano-positioner in a closed-cycle cryostat (Montana instruments, Fusion F2) and all measurements were carried out at about $5 \mathrm{~K}$. The excitation and collection fibers (single-mode fiber, SMF) as well as their associated optics were assembled within a $30 \mathrm{~mm}$ cage system and mounted on the top of the cryostat. Optical excitation was performed off-resonantly by using either a continuous wave or a pulsed $532 \mathrm{~nm}$ laser, which was coupled into the $\mu$ PL setup by a near-infrared-infinity corrected objective (Mitutoyo NIR $50 \times$ ). The PL signal was coupled with a single-mode fiber with $\sim 9 \mu \mathrm{m}$ core size, which acted as a pin-hole to spatially reject stray light from the sample. The combination of the high resolution objective and the single-mode fiber with small mode area allows light emission from a single QD to be addressed. In addition to the $x y z$ nano-positioner, two extra piezoinertia actuators (Thorlabs PIAK10) were installed and they enabled the excitation spot to be moved separately with respect to the collection spot on the hybrid photonic chip. For optical measurements, photoluminescence from either QDs source position or the photonic structure like grating couplers was collected by the same objective and then delivered to the collection fiber. The photoluminescence properties of QDs were analyzed by a high-resolution spectrometer equipped with a charge-coupled device (CCD), and photon correlation measurements were measured by a Hanbury Brown and 
Twiss (HBT) setup consisting of a non-polarizing 50:50 beam splitter, two identical avalanche photodiodes (APDs) and a single-photon counting module (Picoharp 300). For the two-dimensional raster PL scan demonstrated in Figure 2f, we moved the sample in the $x$ and $y$ directions over the microscope objective while keeping the excitation and collection spots overlap.

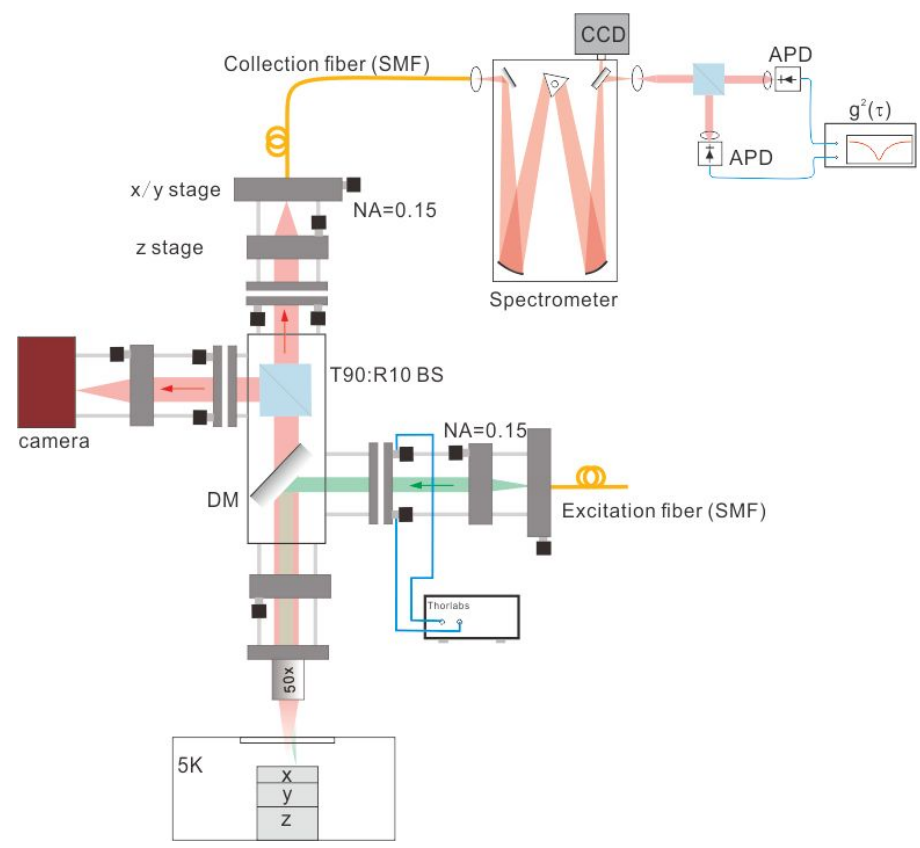

Figure S2. Schematic diagram of the experiment setup. DM - dichromic mirror, SMF - single-mode fiber, NA - numerical aperture.

\section{Design of the grating coupler}

The Bragg-like grating coupler that used to interface the hybrid photonic chip and free space optics consists of three fully-etched rings geometry. With appropriate parameters, the grating coupler is expected to scatter the in-plane propagating electromagnetic field vertically by means of interference effects. According to the similar grating geometry designed for the suspended GaAs photonic waveguides ${ }^{2}, 3$, we chose the initial parameters: $w=\lambda / 2 \mathrm{n}_{\mathrm{GaAs}}=129 \mathrm{~nm}$ and $d=\lambda / 2=450 \mathrm{~nm}$ where $\lambda$ refers to the center wavelength of QD ensemble $(\lambda=890 \mathrm{~nm}), w$ is the ring width and $d$ is the ring separation. FDTD simulation method was then applied to optimize the coupling efficiency and the beam directionality. In our numerical simulations, a dipole source was placed at the center of the GaAs waveguide which was $5 \mu \mathrm{m}$ away from the grating coupler. Figure S3a and b show the coupling efficiency as a function of the grating period and the ring width, respectively. The optimized grating has a period of $470 \mathrm{~nm}$, ring width of $150 \mathrm{~nm}$, which gives a coupling efficiency of $21 \%$ (the blue curve in Figure S3c) for the TE-like mode at the wavelength of $890 \mathrm{~nm}$. Figure $\mathbf{S} 3 \mathrm{~d}$ shows the main electromagnetic field component $\left(E_{\mathrm{y}}\right)$ of the TE-like mode. It should be noted that most of the in-plane propagating light can be scattered vertically within an emission angle of about 20 degree (Figure $1 \mathrm{~d}$ in the main text). In addition, we calculated the 
coupling efficiency for the grating without the beneath gold layer. A coupling efficiency of about $6 \%$ is found and this declined efficiency is mainly due to the leakage of the light into the underlying low refractive polymer material as indicated by the $E_{\mathrm{y}}$ field distribution in Figure S3e. According to the simulations, two channels contribute the main leakage sources for the decline of the out-coupling efficiency. One is the backreflection of the higher-order modes, and the second is the residual forward light propagation. These can be suppressed by using a chirped shallow-etched grating coupler ${ }^{4}$ and using more rings.
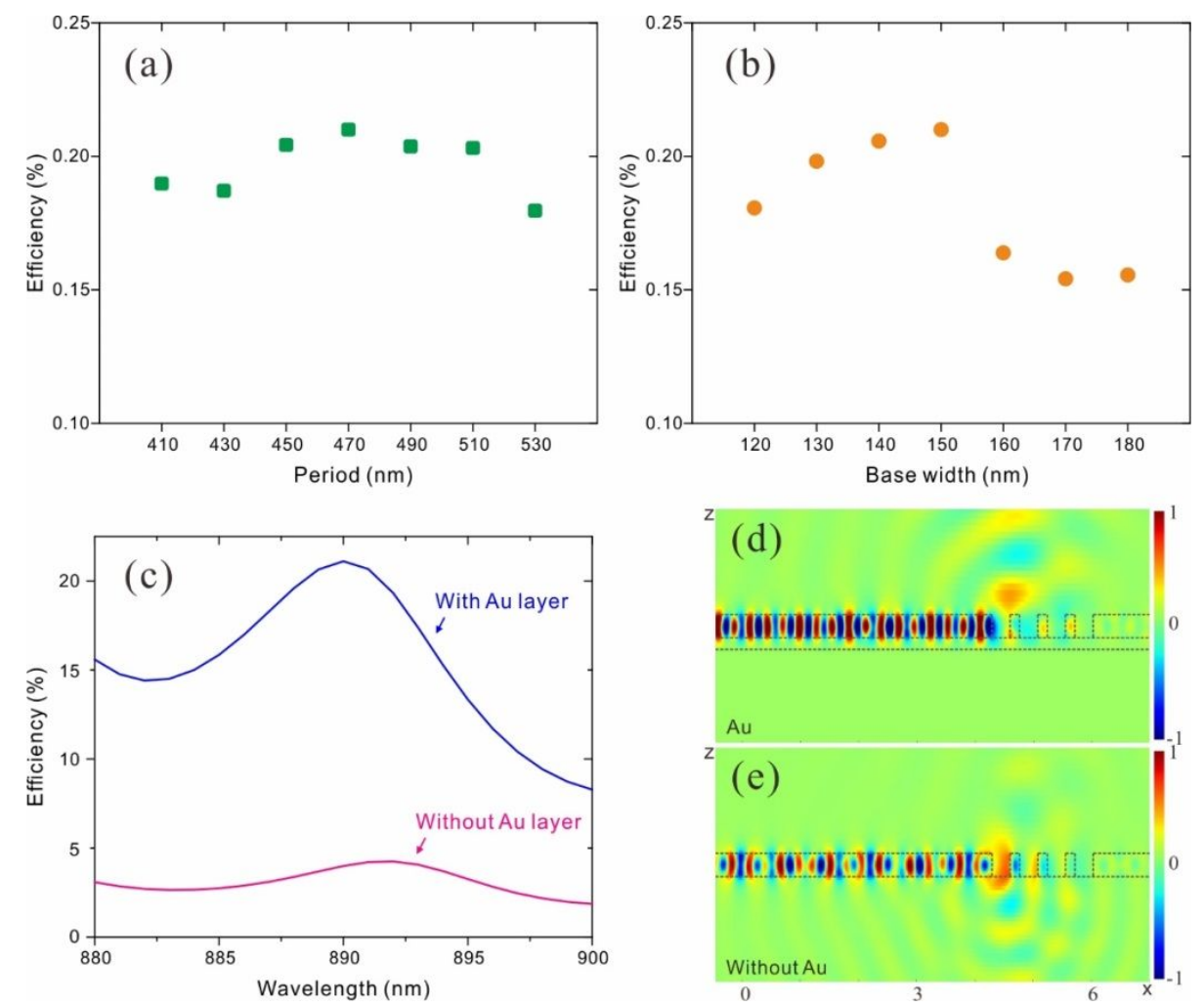

Figure S3. (a) - (b) Dependence of the grating out-coupling efficiency on the period and ring width. (c) Coupling efficiency of the optimized grating coupler with and without the gold layer. (d) - (e) Cross-sectional images of the main electromagnetic $E_{\mathrm{y}}$ field propagating along the waveguide and the grating coupler.

\section{Strain-tuning of individual chip integrated QDs-based single-photon sources: a proposal}

In the present work, we have demonstrated a hybrid piezoelectric-semiconductor quantum photonic chip in which QDs-based single-photon emission can be largely tuned by strain field. Despite the considerable progress, it should be noted that the scalability of the current chip is limited. To achieve scalable on-chip integrated singlephoton sources, strain engineering of individual chip integrated QDs are essentially required. To this end, a long-proposed solution is to fabricate microstructures on the PMN-PT crystal so that independent strain field can be exerted to different QDs simultaneously on one single chip. This will be particularly important for realizing chip 
integrated QDs-based single-photon sources with identical emission energy. Herein we propose one possible way to achieve this goal. Figure $\mathbf{S} 4$ a describes an improved hybrid quantum photonic chip on which GaAs waveguides and a $3 \mathrm{~dB}$ directional coupler are integrated. This GaAs photonic chip can be fabricated and transferred onto to a substrate by using our techniques demonstrated in Figure S1. In order for exerting strain fields to engineer the individually integrated QDs, a thin film of PMN-PT (Figure S4b) could be employed and micro-size strained structures can be fabricated by using focused ion beam milling technique (Figure S4c). Thereafter wet etching is used to remove the bottom metallic gold layer, leaving the patches of the thin PMN-PT unbound on top of the silicon substrate. Next, chromium and gold layer is consecutively deposited on the free-standing PMN-PT patches, and then they are deterministically transferred onto the GaAs photonic platform via a gold-to-gold thermal compression bonding process. In order to obtain adequate transfer accuracy between the PMN-PT patches and the photonic structures, a sub-micron fine placer, e. g. FineTech Lambda, is suggested to be used. Finally, in order to alleviate the clamping effect from the substrate, we fabricate suspended heterogeneous structures. Akin to the fabrication of electromechanical devices such as cantilevers or bridges, nanofabrication technique can be used to fabricate trenches around the heterostructures, and through them wet etching with $\mathrm{KOH}$ solution can be performed to remove the silicon substrate. By suspending the PMN-PT/GaAs/ $/ \mathrm{SiO}_{2}$ heterostructures, the in-plane clamping effect in our proposed hybrid quantum photonic chip can be eliminated and an enhanced piezoelectric performance could be expected. As for the device operation, different voltages can be applied to these strained microstructures so that independent strain fields can be applied to different QDs. With this proposed chip configuration, it is possible to engineer two QDs emitting single photons at the same wavelength, thereby demonstrating the twophoton interference via the $3 \mathrm{~dB}$ directional coupler.
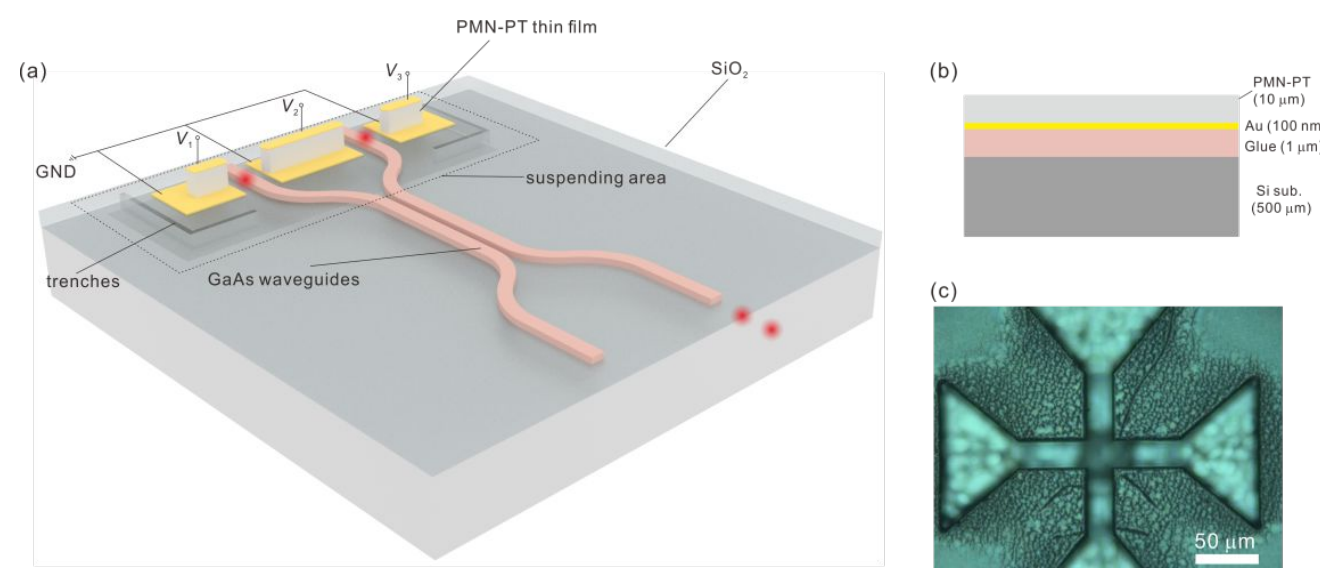

Figure S4. (a) Schematic image of the proposed hybrid quantum photonic chip with a capability of tuning individual QDs by strain fields. (b) Structure of the thin film PMNPT and (c) Strained microstructures fabricated by focus ion beam.

\section{Estimation of QD-to-waveguide coupling efficiency ( $\beta$-factor)}


The coupling efficiency of QD emitters to waveguides represents a key figure of merit for the hybrid quantum photonic chip. To evaluate this coupling efficiency, we carried out an optical simulation by means of a FDTD method. A single QD emitter acting as an in-plane dipole source at $896 \mathrm{~nm}$ was modeled in our simulation. The wavelength was chosen as same as the emission wavelength of the investigated QD shown in Figure 3. Next, the power flux of the QD emission in both propagation directions along the waveguide was computed. Our simulation found that about $26.8 \%$ of light was coupled to the grating that was separated by $5 \mu \mathrm{m}$ from the source, suggesting that a value of $\beta$ $=53.6 \%$ was achieved in our hybrid integrated quantum photonic chip.

Based on the above simulations, we can evaluate the photon coupling efficiency at the source position and at the grating position, respectively. By taking into account the coupling efficiency $(7.2 \%)$ of the grating at $896 \mathrm{~nm}$, we find that about $1.9 \%$ of the light can be collected by the objective at the grating position. In contrast, a higher coupling efficiency of about $6.4 \%$ atop of the source position was found in above optical simulations. This suggests that the collection efficiency at the source position is 3.6 times higher than that of the grating coupler. To confirm this theoretical prediction, we experimentally measured the spectra of the QD in Figure 3 by moving the collection spot from the source position to the grating. The position-dependent peak intensity was obtained and shown in Figure S5. From the plot two bright spots at the source position and at the grating position are found, together with weak count rate along the waveguide due to the light scattering. Interestingly, the photon counts at the source position are about 3 times higher than that of the grating, which is in good agreement with the FDTD simulations.

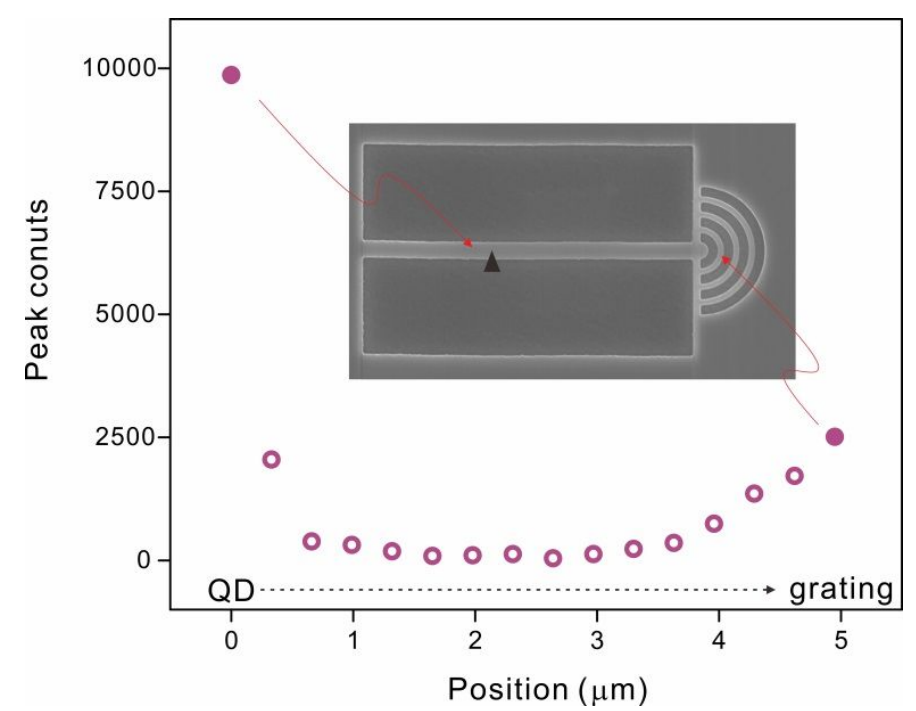

Figure S5. Position dependent peak intensity of the QD embedded in the GaAs waveguide.

\section{Strain tuning results for other QDs that are embedded in a waveguide}


Aside from the investigated QD in the main text, herein we present strain tuning results of the other two waveguide-coupled QDs (denoted by dot 1 and dot 2 respectively) in order to provide more details about our hybrid quantum photonic chip. Note that these two dots were measured by using the same protocols as that of the QD in the main text. These two dots were excited with a laser power of $123 \mathrm{nW}$, and their photon emission was collected on top of the grating coupler. Dynamical tuning these QDs with strain fields was achieved by applying high voltages ranging from $-200 \mathrm{~V}$ to $600 \mathrm{~V}$. As shown in Figure S5, the energy tuning range for the QD emission is found to be $6 \mathrm{meV}$ and $6.6 \mathrm{meV}$, which give a tuning rate of $7.5 \mu \mathrm{eV} / \mathrm{V}$ and $8.25 \mu \mathrm{eV} / \mathrm{V}$ respectively. As compared with the QD in the main text, the tuning rates are slightly lower. This is most probably due to the uneven distribution of strain fields transferred by the PMMA with lower mechanic stiffness. This could be improved by using high mechanic stiffness material such as silicon oxide.
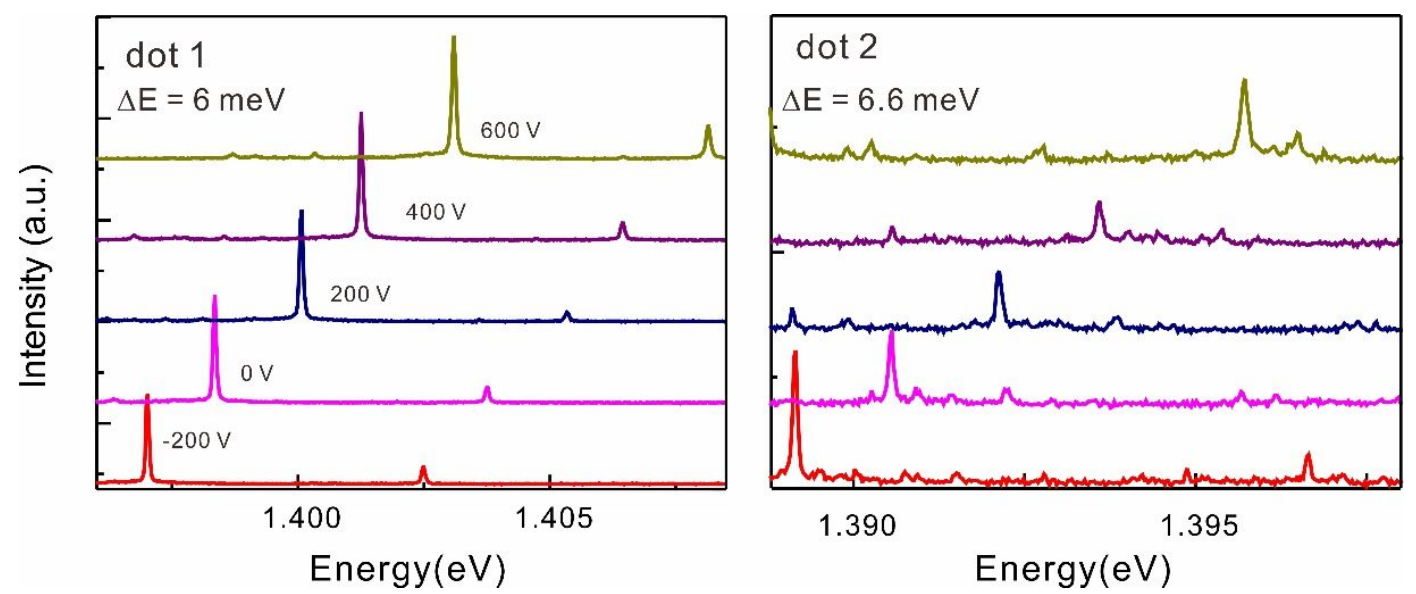

Figure S5. Strain tuning results for other two QDs that are embedded in a waveguide in our hybrid quantum photonic chip.

\section{References}

(1) Zhang, J.; Ding, F.; Zallo, E.; Trotta, R.; Hofer, B.; Han, L.; Kumar, S.; Huo,

Y.; Rastelli, A.; Schmidt, O. G. A nanomembrane-based wavelength-tunable high-speed single-photon-emitting diode. Nano Lett. 2013, 13, 5808-13.

(2) Barth, C.; Wolters, J.; Schell, A. W.; Probst, J.; Schoengen, M.; Lochel, B.;

Kowarik, S.; Benson, O. Miniaturized Bragg-grating couplers for SiN-photonic crystal slabs. Opt. Express 2015, 23, 9803-11. 
(3) Faraon, A.; Fushman, I.; Englund, D.; Stoltz, N.; Petroff, P.; Vuckovic, J. Dipole induced transparency in waveguide coupled photonic crystal cavities. Opt. Express 2008, 16, 12154-62.

(4) Zhou, X.; Kulkova, I.; Lund-Hansen, T.; Hansen, S. L.; Lodahl, P.; Midolo, L. High-efficiency shallow-etched grating on GaAs membranes for quantum photonic applications. Appl. Phys. Lett. 2018, 113, 5. 